\title{
Common genetic variants differentially influence the transition from clinically defined states of fasting glucose metabolism
}

\author{
G. A. Walford • T. Green - B. Neale • T. Isakova • J. I. Rotter • S. F. A. Grant • \\ C. S. Fox • J. S. Pankow • J. G. Wilson • J. B. Meigs $•$ D. S. Siscovick $\cdot$ D. W. Bowden • \\ M. J. Daly • J. C. Florez
}

Received: 13 May 2011 / Accepted: 6 October 2011 / Published online: 29 October 2011

(C) Springer-Verlag 2011

\begin{abstract}
Aims/hypothesis Common genetic variants have been associated with type 2 diabetes. We hypothesised that a subset of these variants may have different effects on the transition from normal fasting glucose (NFG) to impaired fasting glucose (IFG) than on that from IFG to diabetes.

Methods We identified 16 type 2 diabetes risk variants from the Illumina Broad Candidate-gene Association
\end{abstract}

Electronic supplementary material The online version of this article (doi:10.1007/s00125-011-2353-8) contains peer-reviewed but unedited supplementary material, which is available to authorised users.

G. A. Walford · T. Green • B. Neale • M. J. Daly • J. C. Florez $(\bowtie)$

Center for Human Genetic Research, Simches Research

Building - CPZN 5.250, Massachusetts General Hospital,

185 Cambridge Street,

Boston, MA 02114, USA

e-mail: jcflorez@partners.org

G. A. Walford · J. C. Florez

Diabetes Research Center (Diabetes Unit),

Massachusetts General Hospital,

Boston, MA, USA

G. A. Walford · T. Green • B. Neale · M. J. Daly • J. C. Florez Program in Medical and Population Genetics, Broad Institute, Cambridge, MA, USA

G. A. Walford · T. Isakova · J. C. Florez

Department of Medicine, Harvard Medical School,

Boston, MA, USA

T. Isakova

Renal Unit, Massachusetts General Hospital,

Boston, MA, USA

S. F. A. Grant

Center for Applied Genomics, Division of Human Genetics,

Children's Hospital of Philadelphia Research Institute,

Philadelphia, PA, USA
Resource (CARe) array genotyped in 26,576 CARe participants. Participants were categorised at baseline as NFG, IFG or type 2 diabetic $(n=16,465,8,017$ or 2,291 , respectively). Using Cox proportional hazards and likelihood ratio tests (LRTs), we compared rates of progression by genotype for 4,909 (NFG to IFG) and 1,518 (IFG to type 2 diabetes) individuals, respectively. We then performed multinomial regression analyses at baseline, com-

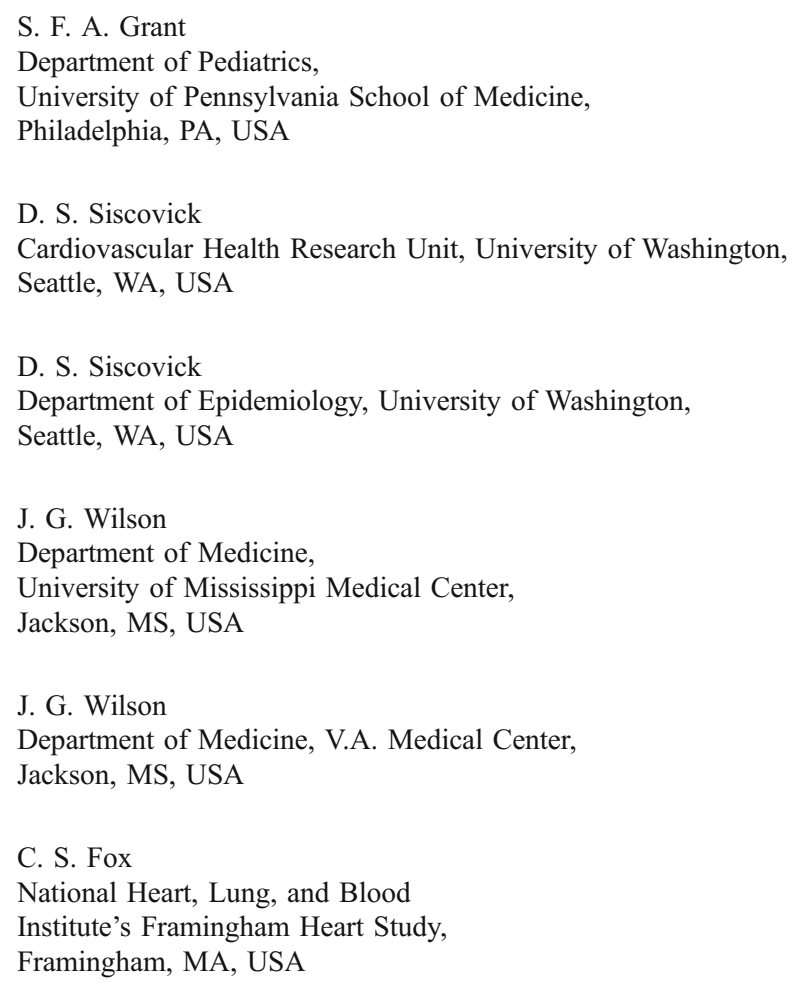


paring the risk of assignment to the NFG, IFG or diabetes groups by genotype.

Results The rate of progression from NFG to IFG was significantly greater in participants carrying the risk allele at MTNRIB $\left(p=1 \times 10^{-4}\right)$, nominally greater at $G C K$ and SLC30A8 $(p<0.05)$ and nominally smaller at IGF2BP2 $(p=0.01)$ than the rate of progression from IFG to diabetes by the LRT. Results of the baseline, multinomial regression model were consistent with these findings.

Conclusions/interpretation Common genetic risk variants at GCK, SLC30A8, IGF2BP2 and MTNR1B influence to different extents the development of IFG and the transition from IFG to type 2 diabetes. Our findings may have implications for understanding the genetic contribution of these variants to the development of IFG and type 2 diabetes.

Keywords Common genetic variants - Diabetes mellitus . Genetics · Glycaemic progression · Impaired fasting glucose $\cdot$ Normal fasting glucose $\cdot$ Single nucleotide polymorphism · Type 2 diabetes

$\begin{array}{ll}\text { Abbreviations } \\ \text { ARIC } & \text { Atherosclerosis Risk in Communities } \\ \text { CARDIA } & \begin{array}{l}\text { Coronary Artery Risk Development in } \\ \text { Young Adults }\end{array} \\ \text { CARe } & \begin{array}{l}\text { Candidate-gene Association Resource } \\ \text { CEU }\end{array} \\ & \begin{array}{l}\text { Centre d'Etude du Polymorphisme (Utah } \\ \text { residents with ancestry from Northern and } \\ \text { Western Europe) }\end{array}\end{array}$

C. S. Fox

Division of Endocrinology, Diabetes, and Hypertension, Brigham and Women's Hospital, Harvard Medical School, Boston, MA, USA

J. B. Meigs

General Medicine Division, Massachusetts General Hospital, Boston, MA, USA

J. B. Meigs

Department of Medicine, Harvard Medical School,

Boston, MA, USA

J. S. Pankow

Division of Epidemiology and Community Health,

University of Minnesota,

Minneapolis, MN, USA

D. W. Bowden

Department of Biochemistry,

Centers for Human Genomics and Diabetes Research,

Wake Forest University School of Medicine,

Winston-Salem, NC, USA

J. I. Rotter

Medical Genetics Institute, Cedars Sinai Medical Center,

Los Angeles, CA, USA

\begin{tabular}{|c|c|}
\hline CHS & Cardiovascular Health Study \\
\hline DIAGRAM & $\begin{array}{l}\text { Diabetes Genetics Replication and } \\
\text { Meta-analysis }\end{array}$ \\
\hline FHS & Framingham Heart Study \\
\hline IBC Chip & Illumina Broad CARe iSelect \\
\hline IFG & Impaired fasting glucose \\
\hline LRT & Likelihood ratio test \\
\hline MAGIC & $\begin{array}{l}\text { Meta-Analysis of Glucose and } \\
\text { Insulin-related Traits Consortium }\end{array}$ \\
\hline MESA & Multi-Ethnic Study of Atherosclerosis \\
\hline NFG & Normal fasting glucose \\
\hline SNP & Single nucleotide polymorphism \\
\hline YRI & Yoruba from Ibadan, Nigeria \\
\hline
\end{tabular}

\section{Introduction}

Impaired fasting glucose (IFG) is a clinically defined prediabetic state, yet only $25 \%$ of individuals with IFG progress to type 2 diabetes mellitus over 3 to 5 years [1]. Evaluation of genetic information may elucidate biological pathways that contribute to the development of IFG and to the clinical transition from IFG to type 2 diabetes.

More and more single nucleotide polymorphisms (SNPs) have been associated unequivocally with increased risk of type 2 diabetes [2]. Recent work by the Meta-Analysis of Glucose and Insulin-related Traits Consortium (MAGIC) and the Diabetes Genetics Replication and Meta-analysis (DIAGRAM) investigators has identified overlap, in crosssectional examination, between loci that influence type 2 diabetes risk and levels of non-diabetic fasting glucose [35]. Longitudinal studies from other groups have identified loci at GCK [6] and MTNR1B [7] that are associated with increased measures of non-diabetic fasting glycaemia and type 2 diabetes risk.

However, results from the MAGIC and DIAGRAM groups have shown that loci associated with a comparable risk of type 2 diabetes have disproportionate associations with elevations in fasting glucose [3, 4]. In addition, the locus with the strongest effect on type 2 diabetes (TCF7L2) has only a modest effect on fasting glucose, whereas one of the loci with the strongest effect on fasting glucose (G6PC2) has a negligible effect on type 2 diabetes [3, 4]. More recently, investigators have demonstrated that loci influencing type 2 diabetes risk and fasting glucose levels at baseline are associated with non-significant increases in fasting glucose over 10 years [8], while others have shown that the association between fasting glucose and genetic variants that influence fasting glucose are stable over time, similarly to age-related changes in non-diabetic individuals [9].

Together, these findings raise the hypothesis that a subset of common type 2 diabetes risk variants may strongly promote the development of non-diabetic elevations in fasting 
glucose, i.e. the transition from normal fasting glucose (NFG) to IFG, but weakly influence the development of type 2 diabetes, i.e. the transition from IFG to type 2 diabetes. To test this hypothesis, we performed analyses within the Candidategene Association Resource (CARe) [10]. This consortium comprises nine National Heart Lung Blood Institute (NHLBI)-supported cohort studies, which have accrued longitudinal clinical measurements, including cardiovascular and glycaemic traits, in approximately 50,000 participants; all participants have been genotyped in a custom-made Illumina Broad CARe iSelect (IBC Chip) array [11].

\section{Methods}

Study participants and clinical characteristics From the nine cohorts within CARe [10], longitudinal fasting glucose measures were available in Atherosclerosis Risk in Communities (ARIC) [12], Coronary Artery Risk Development in Young Adults (CARDIA) [13], Cardiovascular Health Study (CHS) [14], Framingham Heart Study (FHS) [15] and Multi-Ethnic Study of Atherosclerosis (MESA) [16]. Protocols for CARe were approved by local ethics committees and/or institutional review boards of each participating recruitment or analysis site. Informed consent was obtained from all study participants.

Glycaemic phenotypes We used the ADA criteria [17] to categorise participants as NFG $(<5.6 \mathrm{mmol} / \mathrm{l})$, IFG (5.6$6.9 \mathrm{mmol} / \mathrm{l})$ or type 2 diabetic $(\geq 7.0 \mathrm{mmol} / \mathrm{l})$ based on the measurements within CARe. Participants who, despite having a fasting glucose measurement within the expected range, had been otherwise identified at the time of measurement as having diabetes or taking medications to treat diabetes (oral medications or insulin) were included and analysed in the type 2 diabetes group. OGTT results, if available at the time of the fasting measurement, were used only to re-assign participants to the type 2 diabetes group on the basis of a $2 \mathrm{~h}$ OGTT measurement $\geq 11.1 \mathrm{mmol} / \mathrm{l}$.

Measurements Measurements of fasting glucose were performed as previously described in ARIC [18], CARDIA [19], CHS [20], FHS [21, 22] and MESA [23]; all were conducted 8 to $12 \mathrm{~h}$ after the last meal. Follow-up fasting glucose measurements occurred on average at 3, 6 and 9 years after baseline for ARIC, at 3 and 8 years after baseline for CARDIA, at 3 and 7 years after baseline for CHS, at 4, 8, 12 and 16 years after baseline for FHS, and at 2, 4 and 6 years after baseline for MESA.

SNP selection and genotyping Genotyping had been performed previously on the custom-made IBC Chip [11] and the genotype list is publicly available [24]. The IBC chip was designed in 2007 and included type 2 diabetesassociated SNPs identified up until that time (including early access to some findings published later) [25-28]. Tag SNPs were selected as previously described [11]. Approximately 99.5\% concordance was observed against HapMap data, and SNPs failing Hardy-Weinberg equilibrium $(p<0.01)$ were excluded. To optimise capture of the more recently identified type 2 diabetes risk SNPs, we used the SNP Annotation and Proxy Search (SNAP) program [29] to identify the best proxies within the CARe genotype database, with a lower linkage disequilibrium threshold of $r^{2}=0.8$. The linkage disequilibrium was estimated using the Centre d'Etude du Polymorphisme (Utah residents with ancestry from Northern and Western Europe) (CEU) for EuropeanAmerican samples and the Yoruba from Ibadan, Nigeria (YRI) for African-American samples (HapMap release 21 [30]), as used in the design of the IBC chip

Statistical analysis We identified all SNPs or their proxies in CARe that had previously been associated with type 2 diabetes at genome-wide significance $\left(p<5 \times 10^{-8}\right)$. We validated the reported type 2 diabetes associations in CARe by estimating allelic ORs of assignment to the type 2 diabetes versus the NFG groups. We meta-analysed the results separately for European-American and AfricanAmerican participants, using the Cochran-Mantel-Haenszel method for each risk allele and type 2 diabetes versus NFG group allocation at the baseline measurement. Because a detectable association signal with increased type 2 diabetes risk is a necessary prerequisite for statistical comparison of the transition from NFG to IFG with that from IFG to type 2 diabetes, we selected for further analysis only those risk variants that were associated with at least a nominally significant $(p<0.05)$ increase in type 2 diabetes risk in CARe. Power to detect type 2 diabetes risk in the EuropeanAmerican and African-American participants in CARe was determined with the Genetic Power Calculator (http:// ibgwww.colorado.edu/ pshaun/gpc/) [31].

For the 11 risk variants that were associated with increased risk of type 2 diabetes in CARe, we fitted standard Cox regression models to compare the rate of progression for individuals at transition from NFG to IFG (3,836 European-American and 1,073 African-American participant events) and from IFG to type 2 diabetes $(1,060$ European-American and 458 African-American participant events) based on the presence of risk alleles. Individuals who progressed from NFG to type 2 diabetes (109 European-Americans, 80 African-Americans) during a single observation period were excluded from the longitudinal analyses, as it was not possible to parse the rate from NFG to IFG or IFG to type 2 diabetes. Individuals who regressed from IFG to NFG were included in longitudinal analyses examining further progressions to type 2 diabetes 
(i.e. IFG to type 2 diabetes), but were not included in longitudinal analyses examining subsequent NFG to IFG transitions (i.e. return to IFG status). Participants categorised as type 2 diabetes were included in the type 2 diabetes group at all subsequent time points, regardless of their follow-up fasting blood sugar values. Therefore, participants in the type 2 diabetes group at the baseline measurement did not contribute to the longitudinal analyses. All data were analysed together and a covariate for each cohort was included in the analysis. Time to progression was determined by subtracting the participant's age at baseline measurement from age at the follow-up measurement. We calculated HRs for each risk allele, adjusting these analyses for age at baseline and sex.

Because the sample size for transition from NFG to IFG and from IFG to type 2 diabetes differed, we directly compared the effects of type 2 diabetes risk alleles at the two clinical transitions using a likelihood ratio test (LRT). For the LRT, we used a one-degree of freedom $\chi^{2}$ test, which compared the maximum likelihood of a risk allele being associated with the HR averaged across the first and second transitions against a model with the first and second transition HRs. The null hypothesis for the LRT was that the HR of either clinical transition was the same as the average HR of two transitions. A significant finding indicated that the HR at one transition was different from the HR at the other clinical transition. As the IFG group was present in both clinical transitions, we were able to compare the two HRs in a nested model. Intercohort heterogeneity was tested by calculating the maximum likelihood values for the HR of each clinical transition per cohort and comparing each cohort HR with the HR of the other cohorts by a 4 degrees of freedom $\chi^{2}$ analysis.

We then used multinomial logistic regression to examine the association of risk alleles with the NFG versus the IFG groups $(12,480$ and 6,251 European-American participants, and 3,985 and 1,766 African-American participants, respectively) and with the IFG versus the type 2 diabetes groups (6,251 and 1,422 European-American participants, and 1,766 and 869 African-American participants, respectively) at the baseline measurement of fasting glucose in CARe. Participants were grouped according to their baseline measurements, irrespective of later fasting glucose measurements. These analyses generated ORs adjusted for age at baseline and sex; all participants with baseline fasting glucose measurements were included in the analyses. A Wald test was then used to compare the two ORs at the baseline measurement.

\section{Results}

We determined the influence of known type 2 diabetes risk alleles on the transition from NFG to IFG and from IFG to type 2 diabetes in 20,153 European-American and 6,423
African-American CARe participants (Table 1). Categorisation of fasting glucose groups by cohort is provided in the Electronic supplementary material (ESM) Table 1. At the baseline measurement of fasting glucose, 12,480 EuropeanAmerican and 3,985 African-American participants were classified as NFG, 6,251 and 1,766 respectively as IFG, and 1,422 and 869 respectively as type 2 diabetic. There were 1,060 European-American and 458 African-American incident cases of diabetes over $7.7 \pm 2.1$ years $($ mean \pm SD) of follow-up on average. Of the participants with NFG at baseline and follow-up data available, 31\% (3,836 of $12,480)$ of European-Americans and 27\% (1,073 of 3,985) of African-Americans progressed to IFG. Of the participants with IFG and follow-up data available, 15\% (923 of $6,251)$ of European-Americans and 23\% (413 of 1,766) of African-Americans progressed to type 2 diabetes by the last measure of fasting glucose. All participants categorised as type 2 diabetic at baseline were analysed in the type 2 diabetes group at all follow-up measurements. A single OGTT measurement was available for 14,445 participants, and more than one OGTT measurement was available for 9,307 participants. When an OGTT measurement was available at the time of fasting glucose measurement, $4 \%$ of all participants otherwise assigned to the NFG or IFG

Table 1 Demographic data for CARe participants per baseline glycaemic categories

\begin{tabular}{lllll}
\hline Variable per ethnic group & \multicolumn{3}{l}{ Baseline group } & \multirow{2}{*}{ Total } \\
\cline { 2 - 4 } & NFG & IFG & Diabetes & \\
\cline { 2 - 4 } European-American & & & & \\
$n$ & 12,480 & 6,251 & 1,422 & 20,153 \\
NFG to IFG $(n)$ & 3,836 & - & - & 3,836 \\
IFG to type 2 diabetes $(n)$ & 137 & 923 & - & 1,060 \\
Men $(n)$ & 5,027 & 3,442 & 768 & \\
Women $(n)$ & 7,408 & 2,802 & 649 & \\
Sex not specified $(n)$ & 45 & 7 & 5 & \\
FPG (mmol/l) & $5.0 \pm 0.7$ & $6.0 \pm 0.4$ & $10.0 \pm 3.5$ & \\
BMI (kg/m $\left.{ }^{2}\right)$ & $26 \pm 5$ & $28 \pm 5$ & $30 \pm 6$ & \\
Age (years) & $54 \pm 14$ & $59 \pm 11$ & $63 \pm 11$ & \\
African-American & & & & \\
$n$ & 3,985 & 1,766 & 869 & 6,423 \\
NFG to IFG $(n)$ & 1,073 & - & - & 1,073 \\
IFG to type 2 diabetes $(n)$ & 45 & 413 & - & 458 \\
Men $(n)$ & 1,527 & 762 & 342 & \\
Women $(n)$ & 2,458 & 1,004 & 527 & \\
FPG (mmol/l) & $5.0 \pm 1.3$ & $6.0 \pm 0.4$ & $11.3 \pm 4.4$ & \\
BMI (kg/m $\left.{ }^{2}\right)$ & $28 \pm 6$ & $30 \pm 6$ & $32 \pm 6$ & \\
Age (years) & $48 \pm 17$ & $54 \pm 12$ & $58 \pm 9$ & \\
\hline & & & & \\
\hline
\end{tabular}

Values are mean \pm SD unless indicated otherwise

FPG, fasting plasma glucose 
groups $(1,063$ of 24,431$)$ at the first measure of fasting glucose and $1.4 \%$ of all participants otherwise assigned to the NFG or IFG groups (312 of 22,096) at the last measure of fasting glucose had overt diabetes by OGTT; these participants were reclassified as belonging to the type 2 diabetes group to ensure appropriate risk estimates for participants with known diabetes.

Of the 38 published type 2 diabetes signals, we identified 16 risk SNPs or their proxies in the CARe genotype database (Table 2). Based on our pre-specified criteria for inclusion in the study, i.e. demonstrating nominal association with type 2 diabetes in our cohort, we selected 11 SNPs in European-American participants and one in African-American participants for further analyses.

The results of the LRT for the Cox proportional hazards model analyses are provided in Table 3. In EuropeanAmerican participants, those who carried the common type 2 diabetes variant at MTNR1B had a significantly higher risk of transition from NFG to IFG than from IFG to type 2 diabetes during the observed follow-up period $\left(p=1 \times 10^{-4}\right)$. Participants carrying the common risk variants at $G C K$ and SLC30A8 had a nominally higher risk of transition from NFG to IFG than from IFG to type 2 diabetes during the observed follow-up ( $p<0.05$ for all). Participants carrying the risk variant at $I G F 2 B P 2$ had a nominally higher risk of transition from IFG to type 2 diabetes than from NFG to IFG during the observed follow-up $(p=0.01)$. Participants carrying the other genotyped common risk variants had an equal risk of transition from NFG to IFG and from IFG to type 2 diabetes. Interestingly, participants carrying the risk allele at $G C K R$ had a statistically positive OR for transition from NFG to IFG (OR 1.02-1.12, $p=3 \times 10^{-3}$ ), but not for transition from IFG to diabetes $(0.91-1.09, p=0.89)$. Those carrying the risk allele at $T C F 7 L 2$ had a statistically positive OR for transition from NFG to IFG (1.021.13, $\left.p=4 \times 10^{-3}\right)$ and from IFG to diabetes (1.11-1.33, $p=$ $\left.1.5 \times 10^{-5}\right)$. However, in both of the above instances, the association of the risk allele with the NFG to IFG transition was not significantly different from the IFG to diabetes transition in the longitudinal analyses. The test for inter-cohort heterogeneity was unremarkable for all variants in European-American participants $\left(\chi^{2}\right.$ analysis, $p>0.05$ ) (Table 3).

Comparisons between allocation to the IFG versus the NFG group and to the type 2 diabetes versus the IFG group at baseline in European-American participants are displayed in Table 4. The OR for the IFG versus NFG comparison was nominally significant $(p<0.05)$ and greater than 1 for all variants examined, except at WFS1, IGF2BP2 and $K C N Q 1$. The OR for the type 2 diabetes versus IFG comparison was nominally significant and greater than 1 for variants at $T C F 7 L 2, W F S 1$ and IGF2BP2, and nominally significant, but less than 1 at MTNRIB. The Wald test was significant for variants at $G C K, G C K R$ and MTNR1B $(p<0.005$, all indicating a greater effect of the risk allele for the IFG versus NFG comparison than for the type 2 diabetes versus IFG comparison), and at TCF7L2 ( $p<$ 0.001 , indicating a greater effect for the type 2 diabetes

Table 2 Selection of risk variants in the CARe IBC chip

\begin{tabular}{|c|c|c|c|c|c|c|c|c|c|}
\hline Gene region & CARe SNP & Risk allele & $\operatorname{CEU}\left(r^{2}\right)^{\mathrm{a}}$ & YRI $\left(r^{2}\right)^{\mathrm{a}}$ & MAF & OR $(E A)^{b}$ & $p$ value & OR $(\mathrm{AA})^{\mathrm{b}}$ & $p$ value \\
\hline NOTCH2 & rs 835574 & $\mathrm{~T}(\min )$ & 0.9 & - & 0.13 & 1.19 & $5 \times 10^{-3}$ & - & - \\
\hline TCF7L2 & rs7903146 & $\mathrm{T}(\min )$ & 1.0 & 1.00 & 0.29 & 1.40 & $2 \times 10^{-14}$ & 1.33 & $1 \times 10^{-6}$ \\
\hline GCKR & rs780094 & C (maj) & 1.0 & 1.00 & 0.42 & 1.27 & $5 \times 10^{-8}$ & 1.09 & 0.23 \\
\hline$C D K A L 1$ & rs 7754840 & $\mathrm{C}(\min )$ & 1.0 & - & 0.31 & 1.17 & $4 \times 10^{-4}$ & - & - \\
\hline$G C K$ & rs6975024 & $\mathrm{C}(\min )$ & 1.0 & 1.00 & 0.17 & 1.19 & $2 \times 10^{-3}$ & 1.01 & 0.91 \\
\hline KCNJ11 & rs 5215 & $\mathrm{C}(\mathrm{min})$ & 1.0 & 1.00 & 0.37 & 1.09 & 0.06 & 0.98 & 0.85 \\
\hline WFS1 & rs5018647 & $\mathrm{C}$ (maj) & 1.0 & 1.00 & 0.40 & 1.11 & 0.02 & 0.94 & 0.32 \\
\hline HHEX & rs5015480 & C (maj) & 1.0 & - & 0.41 & 1.10 & 0.03 & - & - \\
\hline$I G F 2 B P 2$ & rs4402960 & $\mathrm{T}(\min )$ & 1.0 & 1.00 & 0.32 & 1.20 & $3 \times 10^{-5}$ & 1.11 & 0.06 \\
\hline IRS1 & rs2943634 & $\mathrm{C}$ (maj) & 0.9 & - & 0.33 & 1.07 & 0.14 & 1.03 & 0.56 \\
\hline$K C N Q 1$ & rs 231362 & G (maj) & 1.0 & 1.00 & 0.49 & 1.14 & $2 \times 10^{-3}$ & 1.12 & 0.08 \\
\hline PPARG & rs1801282 & $\mathrm{C}$ (maj) & 1.0 & - & 0.12 & 1.13 & 0.06 & - & - \\
\hline SLC $30 A 8$ & rs 13266634 & $\mathrm{C}$ (maj) & 1.0 & - & 0.32 & 1.18 & $3 \times 10^{-4}$ & - & - \\
\hline$H N F 1 A$ & rs 12427353 & G (maj) & 0.8 & - & 0.20 & 1.04 & 0.41 & - & - \\
\hline MTNRIB & rs 10830963 & $\mathrm{G}(\min )$ & 1.0 & 1.00 & 0.26 & 1.19 & $2 \times 10^{-4}$ & 1.06 & 0.62 \\
\hline$C D K N 2 A / 2 B$ & rs10811661 & $\mathrm{T}$ (maj) & 1.0 & - & 0.18 & 1.11 & 0.06 & - & - \\
\hline
\end{tabular}

AA, African-American; EA, European-American; MAF, minor allele frequency in CARe participants; Maj, major allele; Min, minor allele

${ }^{a}$ Linkage disequilibrium metric in the named HapMap populations for the CARe SNP correlated with the published type 2 diabetes risk allele

${ }^{\mathrm{b}}$ Type 2 diabetes-associated OR in CARe participants as indicated 
Table 3 Longitudinal analysis results for European-American participants

\begin{tabular}{|c|c|c|c|c|c|c|c|c|c|}
\hline \multirow[t]{2}{*}{ Gene region } & \multirow[t]{2}{*}{ CARe SNP } & \multicolumn{2}{|l|}{ NFG to IFG } & \multicolumn{2}{|c|}{ IFG to type 2 diabetes } & \multicolumn{2}{|l|}{ LRT } & \multicolumn{2}{|c|}{ Heterogeneity $^{\mathrm{a}}$} \\
\hline & & $\operatorname{HR}(95 \% \mathrm{CI})^{\mathrm{b}}$ & $p$ value & $\operatorname{HR}(95 \% \mathrm{CI})^{\mathrm{b}}$ & $p$ value & $\chi^{2}$ & $p$ value & $\chi^{2}$ & $p$ value \\
\hline $\mathrm{NOTCH} 2$ & rs835574 & $1.03(0.97,1.11)$ & 0.35 & $0.98(0.86,1.11)$ & 0.73 & 1.11 & 0.29 & 1.04 & 0.90 \\
\hline$T C F 7 L 2$ & rs7903146 & $1.07(1.02,1.13)$ & $4 \times 10^{-3}$ & $1.22(1.11,1.33)$ & $1.5 \times 10^{-5}$ & 3.63 & 0.06 & 2.93 & 0.57 \\
\hline GCKR & rs780094 & $1.07(1.02,1.12)$ & $3 \times 10^{-3}$ & $0.99(0.91,1.09)$ & 0.89 & 1.9 & 0.17 & 1.94 & 0.75 \\
\hline$C D K A L 1$ & rs7754840 & $1.02(0.97,1.07)$ & 0.49 & $1.11(1.02,1.21)$ & 0.02 & 1.53 & 0.22 & 0.48 & 0.98 \\
\hline$G C K$ & rs6975024 & $1.12(1.06,1.19)$ & $1 \times 10^{-4}$ & $0.98(0.88,1.09)$ & 0.68 & 6.56 & 0.01 & 0.32 & 0.99 \\
\hline$W F S 1$ & rs 5018647 & $1.00(0.95,1.05)$ & 0.97 & $1.01(0.93,1.10)$ & 0.80 & 0.33 & 0.57 & 1.37 & 0.85 \\
\hline HHEX & rs5015480 & $0.99(0.95,1.04)$ & 0.82 & $1.05(0.97,1.15)$ & 0.24 & 0.75 & 0.39 & 2.08 & 0.72 \\
\hline$I G F 2 B P 2$ & rs 4402960 & $1.00(0.95,1.05)$ & 0.87 & $1.17(1.07,1.28)$ & $4.4 \times 10^{-4}$ & 6.79 & 0.01 & 1.38 & 0.85 \\
\hline$K C N Q 1$ & rs 231362 & $0.95(0.91,0.99)$ & 0.03 & $1.04(0.95,1.13)$ & 0.40 & 1.55 & 0.21 & 1.39 & 0.85 \\
\hline SLC $30 A 8$ & rs 13266634 & $1.08(1.03,1.14)$ & $2 \times 10^{-3}$ & $0.99(0.90,1.08)$ & 0.78 & 5.08 & 0.02 & 2.72 & 0.61 \\
\hline MTNRIB & rs 10830963 & $1.20(1.15,1.27)$ & $3 \times 10^{-13}$ & $0.98(0.89,1.07)$ & 0.62 & 14.98 & $1 \times 10^{-4}$ & 1.16 & 0.89 \\
\hline
\end{tabular}

${ }^{\text {a }}$ Inter-cohort heterogeneity by risk allele

${ }^{\mathrm{b}}$ From the Cox proportional models

versus IFG comparison than for the IFG versus NFG comparison). The Wald test bordered on nominal significance $(p=0.05)$ for the variant at IGF2BP2 (suggesting a greater effect for the type 2 diabetes versus IFG comparison than for the IFG versus NFG comparison).

We tested the performance of the type 2 diabetes risk allele at TCF $7 L 2$ in longitudinal and multinomial baseline regression models in African-American participants (ESM Table 2). For African-American participants carrying this risk allele, there was an equal rate of transition from NFG to IFG and from IFG to type 2 diabetes. The test for intercohort heterogeneity was unremarkable. In the baseline analysis, the OR for the type 2 diabetes versus IFG comparison was significant $\left(p=6 \times 10^{-5}\right)$ and the Wald test was significant $\left(p=5 \times 10^{-4}\right.$, indicating a greater effect for the type 2 diabetes versus IFG comparison than for the IFG versus NFG comparison).

\section{Discussion}

In the present study, we found that in participants carrying common type 2 diabetes risk alleles at GCK, SLC30A8 and $M T N R 1 B$, the rate of progression from NFG to IFG was higher than that from IFG to type 2 diabetes, while in those carrying the common risk allele at $I G F 2 B P 2$, progression from IFG to type 2 diabetes occurred at a higher rate than progression from NFG to IFG. These longitudinal observations were consistent with those in the baseline multinomial regression model. In the presence of the GCK and MTNRIB

Table 4 Multinomial baseline regression model results for European-American participants

\begin{tabular}{|c|c|c|c|c|c|c|c|}
\hline \multirow[t]{2}{*}{ Gene region } & \multirow[t]{2}{*}{ CARe SNP } & \multicolumn{2}{|c|}{ IFG vs NFG (referent) } & \multicolumn{2}{|c|}{ Type 2 diabetes vs IFG (referent) } & \multicolumn{2}{|c|}{ Wald test } \\
\hline & & OR $(95 \% \mathrm{CI})$ & $p$ value & OR $(95 \% \mathrm{CI})$ & $p$ value & Statistic & $p$ value \\
\hline $\mathrm{NOTCH} 2$ & rs835574 & $1.10(1.03,1.17)$ & 0.01 & $1.02(0.91,1.13)$ & 0.77 & 1.48 & 0.22 \\
\hline TCF $7 L 2$ & rs7903146 & $1.09(1.04,1.15)$ & $4 \times 10^{-4}$ & $1.27(1.18,1.37)$ & $7 \times 10^{-10}$ & 10.67 & 0.001 \\
\hline GCKR & rs780094 & $1.19(1.14,1.25)$ & $8 \times 10^{-14}$ & $1.04(0.97,1.12)$ & 0.30 & 9.5 & 0.002 \\
\hline$C D K A L 1$ & rs 7754840 & $1.09(1.03,1.14)$ & 0.001 & $1.05(0.97,1.13)$ & 0.25 & 0.68 & 0.41 \\
\hline$G C K$ & rs6975024 & $1.23(1.16,1.30)$ & $6 \times 10^{-12}$ & $0.95(0.87,1.05)$ & 0.32 & 20.59 & $6 \times 10^{-6}$ \\
\hline WFS1 & rs5018647 & $1.01(0.96,1.06)$ & 0.69 & $1.09(1.01,1.17)$ & 0.02 & 2.95 & 0.09 \\
\hline HHEX & rs5015480 & $1.08(1.03,1.13)$ & 0.001 & $1.03(0.98,1.14)$ & 0.36 & 1.04 & 0.31 \\
\hline$I G F 2 B P 2$ & rs4402960 & $1.04(0.99,1.09)$ & 0.11 & $1.14(1.06,1.23)$ & 0.001 & 3.86 & 0.05 \\
\hline$K C N Q 1$ & rs231362 & $1.03(0.99,1.08)$ & 0.14 & $1.06(0.98,1.14)$ & 0.13 & 0.26 & 0.61 \\
\hline SLC $30 A 8$ & rs 13266634 & $1.11(1.05,1.16)$ & $5 \times 10^{-5}$ & $1.04(0.96,1.12)$ & 0.35 & 1.84 & 0.17 \\
\hline MTNRIB & rs 10830963 & $1.35(1.28,1.42)$ & $5 \times 10^{-31}$ & $0.88(0.81,0.95)$ & 0.001 & 79.63 & $5 \times 10^{-19}$ \\
\hline
\end{tabular}


risk alleles, the odds of a participant being in the IFG versus the NFG group were greater than the odds of being in the type 2 diabetes versus the IFG group, whereas in the presence of the IGF2BP2 risk allele, the odds of a participant being in the type 2 diabetes versus the IFG group were greater than the odds of being in the IFG versus the NFG group. While participants carrying the risk allele at SL30A8 were significantly more likely to be in the IFG than the NFG group, they were as likely to be in the IFG as in the type 2 diabetes groups in the baseline analyses. These results suggest that the biological effect of type 2 diabetes risk alleles at GCK, SLC30A8 and MTNR1B may be more important for the development of prediabetic fasting hyperglycaemia than for overt diabetes, and that the risk allele at $I G F 2 B P 2$ may be more important for the progression from IFG to type 2 diabetes than for that from NFG to IFG.

Notably, participants carrying the common type 2 diabetes risk alleles at GCKR and TCF7L2 progressed at statistically equal rates from NFG to IFG and from IFG to type 2 diabetes in the longitudinal analyses, suggesting that these risk loci are active in the development of IFG and overt diabetes. However, the ORs in the longitudinal analyses and the results of the baseline multinomial analyses indicate that the GCKR locus may be more associated with the transition from NFG to IFG than with the development of overt diabetes, and that the TCF $7 L 2$ locus may be more associated with the development of overt diabetes from IFG. These discrepancies merit further investigation in European and African populations.

Our study has high statistical power, a large number of participants and longitudinal measurement of fasting glucose over time, as well as using clinically defined measures of glycaemia. Nevertheless, we acknowledge several limitations.

First, relatively few participants in CARe have longitudinal OGTT data, a measure sometimes used in clinical practice for the diagnosis of diabetes and prediabetic states [32, 33]. In a setting of sufficient longitudinal OGTT data and adequate statistical power, a parallel set of analyses using OGTT classification alone and in combination with fasting glucose measurements could be performed to categorise study participants.

Second, of the known type 2 diabetes risk alleles, genotype information and nominal association with type 2 diabetes risk in CARe were available for 16 SNPs only in EuropeanAmerican participants and nine SNPs only in AfricanAmerican participants. This is partly the result of the rapid discovery of new type 2 diabetes loci since the development of the IBC chip in 2007. Updated genotyping arrays and their deployment across these same cohorts might allow more risk alleles to be incorporated into similar analyses in the future.

Third, we had limited power for detection of type 2 diabetes risk in African-American participants in CARe (ESM
Table 3). This stems from the relatively smaller numbers of African-American participants and the less well defined linkage disequilibrium architecture around risk variants in African populations. Replication of these findings in African-American and other non-European populations will be possible in the near future, as improved understanding of type 2 diabetes genetic architecture in multi-ethnic groups and updated genotype arrays incorporating more ancestryinformative SNPs allow the correction of genetic admixture.

While the findings of our study are novel, they expand logically from the existing literature. The type 2 diabetes risk alleles at GCK, SLC30A8 and MTNR1B have been previously associated with an increased cross-sectional and longitudinal risk of type 2 diabetes and elevations in fasting glucose in non-diabetic individuals [3, 4, 6-8, 34-36]. Interestingly, these risk alleles are all associated with agerelated trajectories in fasting glucose, similarly to those in non-diabetic individuals [9]. Our results are consistent with these findings, demonstrating that type 2 diabetes risk loci at GCK, SLC3OA8 and MTNR1B increase the risk of prediabetic fasting glucose levels, but may have minimal influence on the clinical transition from IFG to type 2 diabetes. The type 2 diabetes risk allele at $I G F 2 B P 2$ has also been associated with increased cross-sectional risk of type 2 diabetes and fasting glucose elevations in nondiabetic individuals [37], but its association with longitudinal changes in glucose has not been studied. We observed the strongest effects in our models at the MTNR1B risk allele. Interestingly, this risk allele has recently been shown to be associated with increased fasting glucose values, but decreased $2 \mathrm{~h}$ OGTT values over a 10 year observation period [8], a finding that may be consistent with the seemingly protective influence of the MTNRIB locus for transition from IFG to type 2 diabetes in our study.

The mechanisms by which common variants in $G C K$, $S L C 30 A 8, I G F 2 B P 2$ and MTNR1B exert different degrees of influence on the transition from NFG to IFG and from IFG to type 2 diabetes are unknown. It is notable, however, that mutations in the glucokinase gene impair pancreatic beta cell glucose-sensing, so that individuals with heterozygous mutations develop MODY and classically experience non-progressive hyperglycaemia [38]. Further work may illuminate whether common mutations in $G C K$, as well as SLC30A8 and MTNR1B (which are known to influence beta cell function $[35,36,39])$, and possibly GCKR (which encodes a glucokinase regulatory protein [40]) share common mechanisms in the development of type 2 diabetes. Similarly, variants in $I G F 2 B P 2$ have been associated with reduced beta cell function [5] and reduced insulin secretion [41], but further investigation is needed to understand how this variant has a greater effect on the progression from IFG to overt diabetes than on that from NFG to IFG. 
Conclusions We have found that individuals carrying known type 2 diabetes risk loci at GCK, SLC30A8 and MTNR $1 B$ progressed at a greater rate from NFG to IFG than from IFG to type 2 diabetes over a 7 year period of observation and were more likely to be classified as having IFG than NFG at the baseline observation. Over the same period of time, individuals carrying the risk allele at $I G F 2 B P 2$ progressed at a greater rate from IFG to type 2 diabetes than from NFG to IFG and were more likely to be classified as type 2 diabetic than as having IFG at the baseline observation. The biology by which these loci exert different degrees of influence on these clinical transitions remains speculative and needs to be explored further. These findings, if confirmed, hold implications for a better understanding of the genetic contribution to the development of IFG and type 2 diabetes.

Acknowledgements G.A. Walford (Massachusetts General Hospital) received support from NIH training grant DK007028 and from the Scholars in Clinical Science programme of Harvard Catalyst-The Harvard Clinical and Translational Science Center (award UL1 RR 025758 and financial contributions from Harvard University and its affiliated academic healthcare centres). J.B. Meigs (Massachusetts General Hospital) is supported in part by NIDDK K24 DK080140. J.C. Florez (Massachusetts General Hospital) is supported by a Clinical Scientist Development Award from the Doris Duke Charitable Foundation. The authors wish to acknowledge the support of the National Heart, Lung, and Blood Institute and the contributions of the research institutions, study investigators, field staff and study participants involved in creating this resource for biomedical research. Specific funding information for the nine parent studies contributing to CARe can be found in the ESM text. This study was presented in poster form as a latebreaking abstract at the 2010 American Diabetes Association Scientific Sessions in Orlando, FL, USA.

Contribution statement JCF, GAW and TI were involved with conception and design. JCF, GAW, TG, BN, MJD, JIR, SFAG, CSF, JSP, JGW, JBM, DSS, and DWB were involved with analysis and interpretation of data. GAW, TG, BN, MJD and JCF were involved in drafting the article. GAW, TG, BN, TI, JIR, SFAG, CSF, JSP, JGW, JBM, DSS, DWB, MJD and JCF were involved with critical revisions for important intellectual content. GAW, TG, BN, TI, JIR, SFAG, CSF, JSP, JGW, JBM, DSS, DWB, MJD and JCF gave final approval of the version to be published.

Duality of interest J.B. Meigs currently has research grants from GlaxoSmithKline and serves on a consultancy board for Interleukin Genetics. J.C. Florez has received consulting honoraria from Daiichi-Sankyo and AstraZeneca. The remaining authors declare that there is no duality of interest associated with this manuscript.

\section{References}

1. Nathan DM, Davidson MB, DeFronzo RA et al (2007) Impaired fasting glucose and impaired glucose tolerance: implications for care. Diabetes Care 30:753-759
2. Stolerman ES, Florez JC (2009) Genomics of type 2 diabetes mellitus: implications for the clinician. Nat Rev Endocrinol 5:429-436

3. Prokopenko I, Langenberg C, Florez JC et al (2009) Variants in MTNR1B influence fasting glucose levels. Nat Genet 41:77-81

4. Dupuis J, Langenberg C, Prokopenko I et al (2010) New genetic loci implicated in fasting glucose homeostasis and their impact on type 2 diabetes risk. Nat Genet 42:105-116

5. Voight BF, Scott LJ, Steinthorsdottir V et al (2010) Twelve type 2 diabetes susceptibility loci identified through large-scale association analysis. Nat Genet 42:579-589

6. Vaxillaire M, Veslot J, Dina C et al (2008) Impact of common type 2 diabetes risk polymorphisms in the DESIR prospective study. Diabetes 57:244-254

7. Bouatia-Naji N, Bonnefond A, Cavalcanti-Proenca C et al (2009) A variant near MTNR1B is associated with increased fasting plasma glucose levels and type 2 diabetes risk. Nat Genet 41:89-94

8. Renstrom F, Shungin D, Johansson I et al (2011) Genetic predisposition to long-term nondiabetic deteriorations in glucose homeostasis: ten-year follow-up of the GLACIER Study. Diabetes 60:345-354

9. Jensen AC, Barker A, Kumari M, et al. (2011) Associations of common genetic variants with age-related changes in fasting and postload glucose: evidence from 18 years of follow-up of the Whitehall II Cohort. Diabetes 60:1617-1623

10. CARe website. Available from http://www.broadinstitute.org/ gen analysis/care/index.php/Main Page. Accessed 11 October 2011

11. Keating BJ, Tischfield S, Murray SS et al (2008) Concept, design and implementation of a cardiovascular gene-centric $50 \mathrm{k}$ SNP array for large-scale genomic association studies. PLoS One 3: e3583

12. ARIC website. Available from http://www.cscc.unc.edu/ARIC/. Accessed 11 October 2011

13. CARDIA website. Available from http://www.cardia.dopm.uab.edu/. Accessed 11 October 2011

14. CHS website. Available from http://www.chs-nhlbi.org/. Accessed 11 October 2011

15. FHS website. Available from http://www.framinghamheartstudy.org/. Accessed 11 October 2011

16. MESA website. Available from http://www.mesa-nhlbi.org/ default.aspx. Accessed 11 October 2011

17. Genuth S, Alberti KG, Bennett P et al (2003) Follow-up report on the diagnosis of diabetes mellitus. Diabetes Care 26:3160-3167

18. Folsom AR, Eckfeldt JH, Weitzman S et al (1994) Relation of carotid artery wall thickness to diabetes mellitus, fasting glucose and insulin, body size, and physical activity. Atherosclerosis Risk in Communities (ARIC) Study Investigators. Stroke 25:66-73

19. Folsom AR, Jacobs DR Jr, Wagenknecht LE et al (1996) Increase in fasting insulin and glucose over seven years with increasing weight and inactivity of young adults. The CARDIA Study. Coronary Artery Risk Development in Young Adults. Am J Epidemiol 144:235-246

20. Fried LP, Borhani NO, Enright $P$ et al (1991) The Cardiovascular Health Study: design and rationale. Ann Epidemiol 1:263-276

21. Kannel WB, Feinleib M, McNamara PM, Garrison RJ, Castelli WP (1979) An investigation of coronary heart disease in families. The Framingham Offspring Study. Am J Epidemiol 110:281-290

22. Meigs JB, Nathan DM, Wilson PW, Cupples LA, Singer DE (1998) Metabolic risk factors worsen continuously across the spectrum of nondiabetic glucose tolerance. The Framingham Offspring Study. Ann Intern Med 128:524-533

23. Bild DE, Bluemke DA, Burke GL et al (2002) Multi-ethnic study of atherosclerosis: objectives and design. Am J Epidemiol $156: 871-881$ 
24. CARe Background Information. Available from http://www. broadinstitute.org/gen_analysis/care/index.php/Background Information. Accessed 11 October 2011

25. Sandhu MS, Weedon MN, Fawcett KA et al (2007) Common variants in WFS1 confer risk of type 2 diabetes. Nat Genet 39:951-953

26. Saxena R, Voight BF, Lyssenko V et al (2007) Genome-wide association analysis identifies loci for type 2 diabetes and triglyceride levels. Science 316:1331-1336

27. Winckler W, Weedon MN, Graham RR et al (2007) Evaluation of common variants in the six known maturity-onset diabetes of the young (MODY) genes for association with type 2 diabetes. Diabetes 56:685-693

28. Zeggini E, Weedon MN, Lindgren CM et al (2007) Replication of genome-wide association signals in UK samples reveals risk loci for type 2 diabetes. Science 316:1336-1341

29. Johnson AD, Handsaker RE, Pulit SL, Nizzari MM, O'Donnell CJ, de Bakker PI (2008) SNAP: a web-based tool for identification and annotation of proxy SNPs using HapMap. Bioinformatics 24:2938-2939

30. HapMap website. Available from http://hapmap.ncbi.nlm.nih.gov/ Accessed 11 October 2011

31. Purcell S, Cherny SS, Sham PC (2003) Genetic power calculator: design of linkage and association genetic mapping studies of complex traits. Bioinformatics 19:149-150

32. American Diabetes Association (2009) Standards of medical care in diabetes-2009. Diabetes Care 32(Suppl 1):S13-S61

33. American Diabetes Association (2009) Diagnosis and classification of diabetes mellitus. Diabetes Care 32(Suppl 1):S62-S67
34. Sparso T, Bonnefond A, Andersson E et al (2009) G-allele of intronic rs10830963 in MTNR1B confers increased risk of impaired fasting glycemia and type 2 diabetes through an impaired glucose-stimulated insulin release: studies involving 19,605 Europeans. Diabetes 58:1450-1456

35. Ingelsson E, Langenberg C, Hivert MF et al (2010) Detailed physiologic characterization reveals diverse mechanisms for novel genetic loci regulating glucose and insulin metabolism in humans. Diabetes 59:1266-1275

36. Lyssenko V, Nagorny CL, Erdos MR et al (2009) Common variant in MTNR1B associated with increased risk of type 2 diabetes and impaired early insulin secretion. Nat Genet 41:82-88

37. $\mathrm{Wu} \mathrm{Y,} \mathrm{Li} \mathrm{H,} \mathrm{Loos} \mathrm{RJ} \mathrm{et} \mathrm{al} \mathrm{(2008)} \mathrm{Common} \mathrm{variants} \mathrm{in} \mathrm{CDKAL1,}$ CDKN2A/B, IGF2BP2, SLC30A8, and HHEX/IDE genes are associated with type 2 diabetes and impaired fasting glucose in a Chinese Han population. Diabetes 57:2834-2842

38. Fajans SS, Bell GI, Polonsky KS (2001) Molecular mechanisms and clinical pathophysiology of maturity-onset diabetes of the young. N Engl J Med 345:971-980

39. Staiger H, Machicao F, Schafer SA et al (2008) Polymorphisms within the novel type 2 diabetes risk locus MTNR1B determine beta-cell function. PLoS One 3:e3962

40. Beer NL, Tribble ND, McCulloch LJ et al (2009) The P446L variant in GCKR associated with fasting plasma glucose and triglyceride levels exerts its effect through increased glucokinase activity in liver. Hum Mol Genet 18:4081-4088

41. Groenewoud MJ, Dekker JM, Fritsche A et al (2008) Variants of CDKAL1 and IGF2BP2 affect first-phase insulin secretion during hyperglycaemic clamps. Diabetologia 51:1659-1663 